\title{
Proposing a Pedigree Risk Measurement Strategy: Capturing the Intergenerational Transmission of Antisocial Behavior in a Nationally Representative Sample of Adults
}

\author{
Joseph A. Schwartz, ${ }^{1}$ Eric J. Connolly, ${ }^{2}$ Kevin M. Beaver, ${ }^{3,4}{ }^{3}$ Joseph L. Nedelec, $^{5}$ and Michael G. Vaughn ${ }^{6}$ \\ ${ }^{1}$ School of Criminology and Criminal Justice, University of Nebraska Omaha, Lincoln, NE, USA \\ ${ }^{2}$ Department of Criminal Justice, Pennsylvania State University, Abington, PA, USA \\ ${ }^{3}$ College of Criminology and Criminal Justice, Florida State University, Tallahassee, FL, USA \\ ${ }^{4}$ Center for Social and Humanities Research, King Abdulaziz University, Jeddah, Saudi Arabia \\ ${ }^{5}$ School of Criminal Justice, University of Cincinnati, Cincinnati, OH, USA \\ ${ }^{6}$ School of Social Work, Saint Louis University, St. Louis, MO, USA
}

\begin{abstract}
An impressive literature has revealed that variation in virtually every measurable phenotype is the result of a combination of genetic and environmental influences. Based on these findings, studies that fail to use genetically informed modeling strategies risk model misspecification and biased parameter estimates. Twin- and adoption-based research designs have frequently been used to overcome this limitation. Despite the many advantages of such approaches, many available datasets do not contain samples of twins, siblings or adoptees, making it impossible to utilize these modeling strategies. The current study proposes a measurement strategy for estimating the intergenerational transmission of antisocial behavior (ASB) within a nationally representative sample of singletons using an extended pedigree risk approach that relies on information from first- and second-degree relatives. An evaluation of this approach revealed a pattern of findings that directly aligned with studies examining ASB using more traditional twin- and adoption-based research designs. While the proposed pedigree risk approach is not capable of effectively isolating genetic and environmental influences, this overall alignment in results provides tentative evidence suggesting that the proposed pedigree risk measure effectively captures genetic influences. Future replication studies are necessary as this observation remains preliminary. Whenever possible, more traditional quantitative genetic methodologies should be favored, but the presented strategy remains a viable alternative for more limited samples.
\end{abstract}

- Keywords: antisocial behavior, pedigree risk, genetic predisposition, genetic confounding

One of the most consistent empirical findings reported in the behavioral sciences is that both genetic and environmental influences explain variation in just about every measured phenotype. For example, a sizable and developed literature has estimated the proportion of variance explained by genetic and environmental influences across a wide range of phenotypes, including health outcomes (Johnson \& Krueger, 2005; Maes et al., 1997), patterns of ASB (Baker et al., 2007; Barnes et al., 2011; Schwartz \& Beaver, 2015), various internalizing problems (McGue \& Christensen, 2003; McGuffin et al., 2003; Schwartz et al., 2014), and even entry into a wide range of environments (Kendler \& Baker, 2007). The findings from this literature are so conclusive that when writing the laws of behavior genetics, Turkheimer (2000) acknowledged that '[a]ll human behavioral traits are heritable' (p. 160), an observation that has been echoed subsequently (Carey, 2003; Johnson et al., 2009; Plomin et al., 2013).

This observation is powerful in that it underscores the multifactorial process in the underlying etiology of virtually every phenotype, resulting in serious theoretical and methodological repercussions. While there have been at-

RECEIVED 1 August 2015; ACCEPTED 18 August 2015. First published online 17 September 2015.

ADDRESS FOR CORRESPONDENCE: Joseph A. Schwartz, School of Criminology and Criminal Justice, University of Nebraska at Omaha, 310 Nebraska Hall, 901 N. 17th Street, Lincoln, NE 68588-0561, USA.E-mail: jaschwartz@unomaha.edu 
tempts to address such theoretical implications (Harris, 2009; Moffitt, 1993), far more effort has been devoted to the methodological implications (Carey, 2003; Neale \& Cardon, 1992; Plomin et al., 2013). Due to the salience of both genetic and environmental influences in the etiological development of behavioral phenotypes, great effort has been devoted to designing research methodologies that account for both sets of influences (Carey, 2003; DeFries \& Fulker, 1985; Neale \& Cardon, 1992; Plomin et al., 2013). Such methodologies aim to effectively control genetic influences (and typically shared environmental influences) in an effort to isolate the potential effect of an observed environmental influence on the outcome of interest. Failing to properly control for genetic influences on the outcome of interest may result in model misspecification and biased estimates stemming from genetic confounding (Harden et al., 2008; Johnson et al., 2009; McGue et al., 2010).

A number of alternative strategies have been developed, but the most common approaches used to control for genetic confounding continue to utilize twin and/or sibling research designs. While these studies are subject to a series of underlying assumptions, the results of a line of quantitative studies have revealed that such assumptions are robust and the resulting heritability estimates flowing from such designs are accurate (for an overview, see Barnes et al., 2014). Previous research has also indicated that findings from twin studies generalize to larger populations (Barnes \& Boutwell, 2013) and has documented an increase in the overall number of available twin/sibling samples (Hur \& Craig, 2013). However, many available datasets do not contain twin or sibling subsamples. While alternative methodological approaches have been developed (e.g., the adoption-based design), these approaches still rely on the use of specialty samples, effectively limiting the overall number of samples that can be used to estimate genetically informed models.

In an effort to overcome this limitation, additional methodological approaches have been developed that allow for the direct estimation of genetic influences within samples of singletons. More specifically, genome-wide association studies (GWAS), and more recently, genetic complex trait analysis (GCTA) utilize datasets containing thousands of individuals and hundreds of thousands of single nucleotide polymorphisms (SNPs) in an effort to estimate genetic influences from measured genotypes (Plomin et al., 2013; Purcell et al., 2007; Yang et al., 2011). The obvious advantage of these approaches is the ability to estimate genetic influences without the use of a twin or adoptee sample. However, GWAS and GCTA research designs also have strict data requirements. For example, GCTA studies require relatively large samples due to low levels of genetic variation across respondents. In addition, despite significant increases in the number of samples containing information on the hundreds of thousands of SNPs required by GWAS and GCTA studies (Haberstick et al., 2014; Trzaskowski et al., 2013), such samples are still relatively rare.
Despite tremendous methodological advancement in estimating genetic influences and properly accounting for such influences in multivariate models, the availability of appropriate samples remains restricted, particularly for disciplines such as sociology, epidemiology, and criminology. These data limitations have been acknowledged in fields that are interested in examining phenotypes that have been found to be under significant genetic and environmental influence. For example, epidemiologists have developed sibling recurrence risk (SRR) scores to estimate an individual's susceptibility to a given disease based on the prevalence of the disease among genetically related kin, most commonly, siblings (Graubard \& Sirken, 2011; Guo, 1998; Risch, 1990a, 1990b, 1990c). While this method requires information from more than one family member per household, such information can be provided by a single household member. For example, respondents can be asked to report the overall number of their full siblings, and the number of their full siblings who are affected by the disorder in question. Using this information, along with similar questions regarding other family members such as parents or grandparents, SRR scores are calculated as the ratio of the proportion of affected individuals (also referred to as probands) among all siblings to the population prevalence of the examined disorder (for a more comprehensive overview of SRR measures and the necessary formulas see Graubard \& Sirken, 2011). SRR ratios greater than one indicate that various family-level influences (including genetic influences) significantly contribute to the examined disorder (Wallace et al., 2003).

The primary limitation of this particular approach is that the resulting SRR score reflects genetic risk for the full sample and does not vary between families or individuals. This limitation is a direct function of the overall purpose of the SRR, which is simply to estimate whether a given disease has a genetic basis. Because of the lack of individual variation in the SRR, this procedure cannot be used to effectively control for the intergenerational transmission of a given outcome and minimize the potential of genetic confounding. Despite this limitation, the underlying logic of such procedures may provide an interesting avenue of inquiry. More specifically, it remains possible that measures of the prevalence of a given phenotype across family members, reported by a single family member, can potentially be used to create a latent measure of intergenerational risk for that same phenotype. The current study aims to explore this potential alternative measurement strategy using data from the National Epidemiologic Survey of Alcohol and Related Conditions (NESARC). We specifically focus on the construction of an extended pedigree risk approach of ASB based on information regarding the intergenerational transmission and concentration of ASB across firstand second-degree relatives.

ASB was selected as the phenotype of interest for two related reasons. First, ASB is one of the most commonly 
examined behavioral phenotypes, with over 100 studies examining genetic, shared environmental, and non-shared environmental influences (Moffitt, 2005). Second, and directly in line with the first point, four separate meta-analyses have examined the findings of this vast literature and have converged to indicate that approximately $50 \%$ of the overall variance in ASB is explained by genetic influences (Ferguson, 2010; Mason \& Frick, 1994; Miles \& Carey, 1997; Rhee \& Waldman, 2002). Based on this consistency in findings, ASB represents an ideal candidate phenotype for the current investigation. Findings indicating that the ASB pedigree risk factor explains approximately $50 \%$ of the variance in ASB would directly align with one of the most consistent and robust findings in quantitative genetic research. In this way, the pattern of results identified in the extant literature represents a measure of criterion validity with which the results from the current study can be compared.

\section{Methods}

\section{Data}

Data for the current study were drawn from the first wave of the NESARC, which consists of a nationally representative sample of 43,093 adults aged 18 or older from all 50 U.S. states and the District of Columbia, collected between 2001 and 2002 (Grant et al., 2003). A second wave of data was collected between 2004 and 2005, and 34,653 of the respondents from the first wave were re-interviewed $(\mathrm{Na}-$ tional Institute of Alcohol Abuse and Alcoholism, 2010). The NESARC project was carried out under the supervision of the National Institute on Alcohol Abuse and Alcoholism (NIAAA) and used a multistage stratified sampling design. The first stage of the sampling procedure drew from a sampling frame that consisted of all housing units included in the Census 2000/2001 Supplementary Survey (C2SS). Self-representing primary sampling units (PSUs) were selected with certainty, and a probability sample of non-self-representing PSUs was also included. For the second stage of the sampling procedure, housing units within each PSU were systematically selected. Importantly, Hispanic and African-American housing units were selected at a higher probability than other housing units. The third and final stage of the sampling procedure consisted of the selection of one individual per household. Individuals over the age of 18 residing within each household were randomly selected prior to each conducted interview. Importantly, young adults between the ages of 18 and 24 were oversampled in an effort to obtain additional information on the transition from adolescence to young adulthood. Due to clustering resulting from the sampling procedures employed in the NESARC, analyses that assume simple random sampling tend to yield overinflated standard errors (Grant et al., 2003). In an effort to overcome this limitation, the NESARC research team has created sample weights and cluster variables that properly adjust standard errors (for a more detailed overview of the creation of the NESARC sample weights, see Grant et al., 2003). All analyses performed in the current study were estimated using the proper sample weights and cluster variables provided by the NESARC research team.

Interviews were carried out by trained U.S. Census Bureau Field representatives and were completed using a computer-based survey instrument. While one of the primary goals of the NESARC was to estimate the prevalence of alcohol use in the United States, the project also sought to achieve other, broader goals that required the collection of a wide range of additional measures cutting across multiple domains of development. For example, the survey instrument included the Alcohol Use Disorder and Associated Disability Interview Schedule - DSM-IV Version (AUDADIS-IV), which is a diagnostic interview instrument used for the diagnosis of a large number of disorders based on criteria specified in the Diagnostic and Statistical Manual of Mental Disorders, Fourth Edition (DSM-IV; Grant et al., 2001). The survey instrument also included items related to ASB, family problems, current socioeconomic status, physical health, and victimization. The study achieved high response rates with a household response rate of $89 \%$, an individual response rate of $93 \%$, and an overall Wave 1 response rate of $81 \%$. All respondents provided informed consent and the research plan was approved by the U.S. Census Bureau and the U.S. Office of Management and Budget.

\section{Measures}

\section{Antisocial Behavior Measures}

During Wave 1 interviews, respondents were asked 33 questions tapping multiple forms of ASB and directly in line with criteria specified in the DSM-IV. The complete list of all 33 items are indexed in the online supplement and includes items that ask respondents about skipping school, being homeless for more than a month, vandalism, arson, shoplifting, using a weapon in a fight, sexual assault, and physical assault. For each item, respondents were asked three additional questions. First, respondents were asked whether they ever engaged in the incident. Second, respondents were asked whether the same incident occurred before they were 15 years old. Third, respondents were asked whether they engaged in that same incident since they were 15 years old. ${ }^{1}$ For all questions, responses were coded dichotomously where $0=$ no and $1=$ yes. The measurement models used to generate the ASB (and pedigree risk) factors are described in detail below.

\section{Pedigree Risk Measure}

Immediately following the ASB section of the Wave 1 questionnaires, respondents were asked a series of questions regarding the prevalence of ASB within their family. This section consisted of two separate types of questions aimed at 


\section{TABLE 1}

Mean Number of Relatives within Each Examined Group

\begin{tabular}{lllll}
\hline & Mean & SD & Min-Max & $N$ \\
\hline Number of brothers & 1.53 & 1.66 & $0-20$ & 41,979 \\
Number of sisters & 1.46 & 1.57 & $0-20$ & 42,010 \\
Number of aunts (maternal) & 1.96 & 1.88 & $0-20$ & 39,459 \\
Number of aunts (paternal) & 1.81 & 1.76 & $0-20$ & 37,155 \\
Number of uncles (maternal) & 1.96 & 1.85 & $0-19$ & 39,205 \\
Number of uncles (paternal) & 2.07 & 2.03 & $0-20$ & 36,794 \\
\hline
\end{tabular}

Note: Means and standard deviations calculated using sample weights and cluster variables.

tapping family history of ASB. The first type of question was aimed at estimating the number of family members within each family. Respondents were asked to report the number of their full brothers and sisters who lived to be at least 10 years old, along with the total number of both maternal and paternal aunts and uncles who were in their families. ${ }^{2}$ The weighted average number of relatives within each category (along with standard deviations, maximum and minimum values, and sample sizes) is provided in Table 1.

The second type of question used to measure the concentration of ASB within families asked each respondent whether a given member of their family had behavior problems. For example, respondents were asked whether their blood/natural mother ever had behavior problems. These questions were repeated for a total of six relatives: mother, father, maternal grandfather, maternal grandmother, paternal grandmother, and paternal grandfather. Responses to questions about a specific family member were coded dichotomously such that $0=$ no and $1=$ yes. For questions asking about a group of relatives, respondents were asked to report the number of those particular relatives who had behavior problems. For example, respondents were asked to report the number of full sisters who ever had behavior problems. In total, respondents provided information for six additional sets of relatives: full sisters, full brothers, maternal aunts, maternal uncles, paternal aunts, and paternal uncles.

In an effort to better assess the concentration of affected relatives in each family, the sibling, aunt, and uncle risk measures reflect the proportion of affected relatives within these subgroups. Relying on measures indicating the total number of relatives within each subgroup, the proportional family risk measures were coded categorically such that $0=$ no affected relatives, $1=$ up to $25 \%$ of all relatives affected, $2=$ more than $25 \%$ but less than half of all relatives affected, $3=$ more than half but less than $75 \%$ of all relatives affected, and $4=75 \%$ or more of all relatives affected. In total, five family risk measures were used in the current study: parent risk, grandparent risk, sibling risk, aunt risk, and uncle risk. While the categorical measures applied to the sibling, aunt, and uncle subgroups, the parent and grandparent risk measures simply indicate the number of parents or grandparents with reported behavior problems. The frequencies and percentages (along with the frequencies for each group) for all

\section{TABLE 2}

Frequencies and Percentages of Relatives with Behavior Problems

\begin{tabular}{|c|c|c|}
\hline & Frequency & Percentag \\
\hline \multicolumn{3}{|l|}{ Parents } \\
\hline Neither parent & 35,063 & $91.41 \%$ \\
\hline One parent & 2,548 & $6.97 \%$ \\
\hline Both parents & 633 & $1.62 \%$ \\
\hline Group frequency & \multicolumn{2}{|c|}{38,217} \\
\hline \multicolumn{3}{|l|}{ Grandparents } \\
\hline No grandparents & 29,028 & $95.91 \%$ \\
\hline One grandparent & 798 & $2.91 \%$ \\
\hline Two grandparents & 224 & $0.80 \%$ \\
\hline Three grandparents & 55 & $0.20 \%$ \\
\hline Four grandparents & 43 & $0.17 \%$ \\
\hline Group frequency & \multicolumn{2}{|c|}{30,148} \\
\hline \multicolumn{3}{|l|}{ Siblings } \\
\hline No siblings & 30,570 & $87.16 \%$ \\
\hline$\leq 25 \%$ of all siblings & 1,063 & $2.84 \%$ \\
\hline$>25 \%$ and $\leq 50 \%$ of all siblings & 1,620 & $4.79 \%$ \\
\hline$>50 \%$ and $\leq 75 \%$ of all siblings & 483 & $1.31 \%$ \\
\hline All siblings & 1,291 & $3.90 \%$ \\
\hline Group frequency & \multicolumn{2}{|c|}{35,027} \\
\hline \multicolumn{3}{|l|}{ Aunts } \\
\hline No aunts & 26,914 & $94.30 \%$ \\
\hline$\leq 25 \%$ of all aunts & 454 & $1.51 \%$ \\
\hline$>25 \%$ and $\leq 50 \%$ of all aunts & 618 & $2.17 \%$ \\
\hline$>50 \%$ and $\leq 75 \%$ of all aunts & 253 & $0.89 \%$ \\
\hline All aunts & 301 & $1.13 \%$ \\
\hline Group frequency & \multicolumn{2}{|c|}{28,540} \\
\hline \multicolumn{3}{|l|}{ Uncles } \\
\hline No uncles & 24,972 & $89.30 \%$ \\
\hline$\leq 25 \%$ of all uncles & 819 & $2.91 \%$ \\
\hline$>25 \%$ and $\leq 50 \%$ of all uncles & 1,099 & $4.16 \%$ \\
\hline$>50 \%$ and $\leq 75 \%$ of all uncles & 406 & $1.37 \%$ \\
\hline All uncles & 584 & $2.26 \%$ \\
\hline Group frequency & \multicolumn{2}{|c|}{27,880} \\
\hline
\end{tabular}

family risk measures are presented in Table 2 . The creation of the pedigree risk factor is described in detail below.

\section{Plan of Analysis}

The plan of analysis consisted of three interconnected steps that are all presented graphically in Figure 1. First, a series of measurement models were estimated to specify the ASB factor. Exploratory factor analysis (EFA) was used to elucidate the factor structure of the ASB items. A total of three EFA models were estimated: the first included all lifetime ASB items, the second included items tapping early onset ASB (occurring before age 15), and the final model examined the ASB items tapping adult onset ASB (occurring since age 15). In line with previous research (e.g., Beaver et al., 2013), three goodness of fit indices were used to evaluate model fit - the comparative fit index (CFI), the Tucker-Lewis Index (TLI) and the root mean square error of approximation (RMSEA). Previous studies have revealed that values greater than 0.90 on the CFI and TLI indicate reasonably good fit and values greater than 0.95 indicate a close fit $(\mathrm{Hu}$ \& Bentler, 1999). In addition, RMSEA values up to 0.08 indicate reasonable fit, while values below 0.05 indicate a close fit (Byrne, 2012; Hu \& Bentler, 1999). Based on the results of the EFA models, confirmatory factor analysis (CFA) was used to further specify the subfactors that comprised 


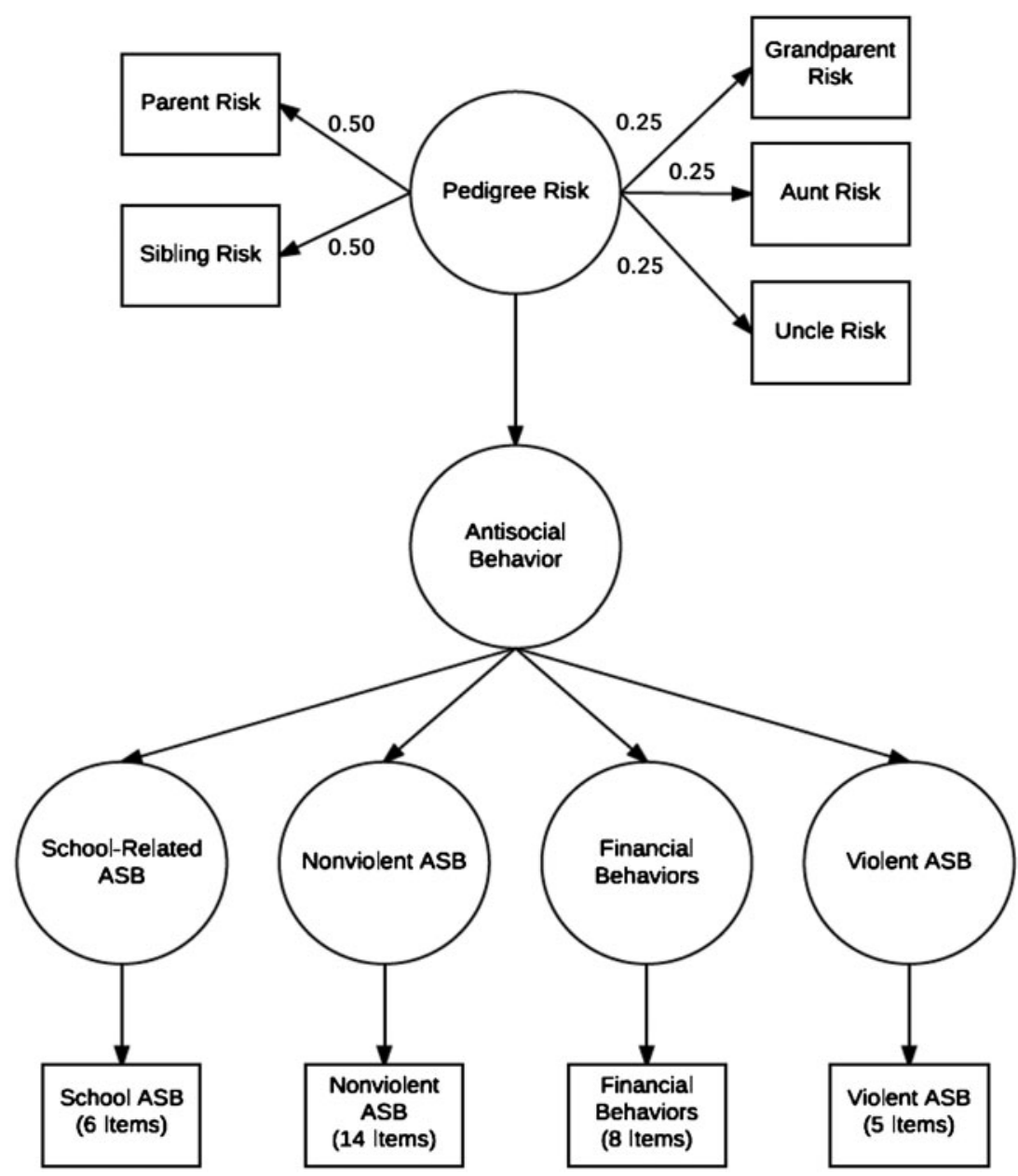

\section{FIGURE 1}

Structural equation model for the pedigree risk measure.

Note: The top half of the path diagram displays the measurement model used to create the pedigree risk factor. The path estimates included in the figure indicate that the factor loadings for first-degree relatives were fixed to 0.50 and the loadings for second-degree relatives were fixed to $\mathbf{0 . 2 5}$. The bottom half of the model displays the measurement model used to create the antisocial behavior factor. In an effort to more clearly display the model, the individual indicators used to create the ASB factors are represented with a single rectangle.

each of the three ASB measures. Finally, the identified subfactors were included in a second-order CFA to identify the higher-order factors of lifetime, early onset, and adult onset ASB. The CFA is presented in the bottom half of Figure 1. Importantly, due to the large number of indicator variables included in the model, the indicators are presented based on the results of the EFA.

The second step of the analysis is presented in the top half of Figure 1 and involved the estimation of CFA to create a latent measure of pedigree risk using the parent, grandparent, sibling, aunt, and uncle risk indicators. In an effort to account for the proportion of shared genetic material between each respondent and members of their extended family, pedigree risk was measured in multiple ways, all of which are described in more detail below. The third and final step in the analysis was aimed at estimating the proportion of variance (estimated as $R^{2}$ coefficients) in each of the three latent ASB measures explained by the pedigree risk factor. Based on the convergence of findings from a substantial number of studies employing twin- and adoption-based research designs to estimate the genetic and environmental influences on ASB (Ferguson, 2010; Mason \& Frick, 1994; Miles \& Carey, 1997; Moffitt, 2005; Rhee \& Waldman, 2002), this stage of the analysis was intended to assess the validity of the extended pedigree risk approach. More specifically, a pattern of results from this stage of the analysis that directly aligns with the extant literature would provide preliminary support for this alternative approach. Due to the well-replicated and substantial differences in ASB patterns between males and females (Ellis \& Walsh, 2000; Leonard, 1995; Martel, 2013), all regression models were estimated for the full sample, and then using sex-restricted subsamples. All analyses in the current study were performed using the statistical software program Mplus version 7.2 (Muthén 
\& Muthén, 2013). Due to the presence of dichotomous and categorical indicator variables, all models were estimated using a robust weighted least squares estimator (WLSMV). Missing data were imputed using a modified version of full information maximum likelihood (FIML) estimation (for more information, see Muthén \& Muthén, 2013, pp. 7-8).

\section{Results}

\section{Antisocial Behavior}

The first step in the analysis was to estimate a series of measurement models to specify the latent ASB measures. The ASB measures were created using three interrelated steps. First, EFA was used to elucidate the factor structure of the ASB items. The results of the three EFA models revealed a similar pattern of results with the models examining lifetime ASB $(\mathrm{CFI}=0.982$, TLI $=0.976$, RMSEA $=0.038)$, early onset ASB $(\mathrm{CFI}=0.991$, TLI $=0.988$, RMSEA $=0.005)$, and adult onset ASB (CFI $=0.984$, TLI $=0.978$, RMSEA $=0.012$ ), all revealing a four-factor solution. ${ }^{3}$ Due to the similarity in the factor structures, only the EFA results for the lifetime ASB measures are presented in the online supplement. The first factor appears to tap school-related ASB items, including cutting class, staying out late, being frequently absent from school, and quitting a school program. The second factor appears to tap non-violent ASB, including having a driver's license revoked, destroying someone else's property, shoplifting, and forging a signature. The third factor taps problematic financial behaviors, including quitting a job without having another lined up, being homeless for over a month, and failing to pay off debts. The fourth and final factor taps more violent criminal behaviors, including bullying, sexual assault, using a weapon in a fight, and domestic violence.

The second step in the creation of the ASB measure involved the use of CFA to further specify each of the four factors identified in the previous step. More specifically, six items were used to create the school-related ASB subfactor, 14 items were used to create the non-violent ASB subfactor, five items were used to create the problematic financial behaviors subfactor, and eight items were used to create the violent ASB subfactor. Each of the items used to specify the four subfactors are identified in the online supplement, wherein the bolded factor loadings indicate that the item was used to specify the corresponding subfactor. The results of the CFA models directly corresponded with the results of the EFA models and indicate a close fit to the data for the lifetime $(\mathrm{CFI}=0.969$, TLI $=0.966$, RMSEA $=0.021)$, early onset $(\mathrm{CFI}=0.975, \mathrm{TLI}=0.973$, RMSEA $=0.006)$, and adult onset (CFI $=0.972$, TLI $=0.971$, RMSEA $=$ 0.012 ) items. In an effort to capture a more global measure of ASB, the third and final step in the creation of the ASB measures involved the estimation of second-order CFA wherein each of the four subfactors were used as indicators of a higher order factor of ASB. Once again, the results of the models examining the lifetime $(\mathrm{CFI}=0.967$, $\mathrm{TLI}=$ 0.965$, RMSEA $=0.021)$, early onset $(\mathrm{CFI}=0.975$, TLI $=$ 0.973 , RMSEA $=0.006)$, and adult onset $(\mathrm{CFI}=0.974$, TLI $=0.971$, RMSEA $=0.012$ ) measures closely aligned and revealed a close fit to the data.

\section{Pedigree Risk}

The pedigree risk measure was created using three sets of CFA models aimed at specifying the underlying factor structure of the parent, grandparent, sibling, aunt, and uncle risk indicators, but also taking into account levels of genetic relatedness between the respondent and each family member. First, a single factor model in which all of the family risk indicators were allowed to freely load on the pedigree risk factor was estimated. While the model provided a close fit to the data $(\mathrm{CFI}=992, \mathrm{TLI}=0.985$; $\mathrm{RMSEA}=0.028)$, this particular measurement strategy does not take into account levels of shared genetic material between the respondent and each relative category. In an effort to address this limitation, a second CFA was estimated in which the factor loadings for first-degree relative (e.g., parents and siblings) indicators and second-degree relative (e.g., grandparents, aunts, and uncles) indicators were constrained to equality. The more restricted model fit the data closely $(\mathrm{CFI}=0.991$, TLI $=$ 0.989; RMSEA $=0.024$ ) but also worsened overall model fit $\left(\Delta \chi^{2}=60.15, p<.001\right)$. Finally, a third model was estimated in which the factor loadings for first-degree family risk indicators were fixed to 0.50 and the loadings for second-degree family indicators were fixed to 0.25 to more directly reflect additive genetic theory. The model fit the data poorly $(\mathrm{CFI}=0.332$, TLI $=0.332$; $\mathrm{RMSEA}=$ $0.181)$ and significantly worsened overall fit $\left(\Delta \chi^{2}=\right.$ 4,713.29, $p<.001)$.

Despite the poor fit of the third CFA model, all three measurement strategies were retained in the subsequent regression analyses. This decision was made for three reasons. First, the overall model fit of the subsequent SEMs containing the third pedigree risk variable indicated an acceptable fit to the data (the CFI ranged between 0.912 and 0.949 , the TLI ranged between 0.907 and 0.948 , and the RMSEA ranged between 0.011 and 0.016 ). These findings indicate that while the most restrictive pedigree risk CFA model may not fit the data well, the larger SEM containing the pedigree risk CFA model provides a much closer fit to the data. Second, the decision to fix the factor loadings at 0.50 for first-degree relatives and 0.25 for second-degree relatives is directly rooted in additive genetic theory, providing a strong theoretical justification for this particular measurement strategy. In addition, more traditional biometric modeling strategies employ a similar measurement technique, in which the covariance of a given phenotype between a kinship pair is fixed to reflect the level of genetic relatedness between the pair (e.g., 1.00 for monozygotic twins and 0.50 for dizygotic twins and full siblings). Third, fixing the factor loadings to reflect levels of genetic 
relatedness should truncate overall variance in the resulting latent factor. A reduction in overall levels of variance would likely reflect at least the partial removal of environmental sources of influence on ASB and a better isolation of genetic influences. While this modeling strategy would not necessarily rule out any sources of shared environmental influence, the most restrictive measurement strategy should result in a latent pedigree risk measure that contains less variance attributable to shared environmental influence relative to the less restrictive measurement models.

\section{Regression Models}

The next step in the analysis involved estimating a series of regression models to estimate the proportion of variance in ASB explained by the latent pedigree risk measure. Importantly, models were estimated for all three ASB factors. The results of the regression models are presented in Table 3. The first set of models regressed each of the three latent ASB measures on the pedigree risk factor in which the family risk indicator loadings were freely estimated. The results are presented in the first set of columns and include unstandardized and standardized path estimates, the accompanying standard errors, and the proportion of variance explained presented as an $R^{2}$ estimate for each of the examined ASB outcomes. The results indicate that the pedigree risk factor explained approximately $39 \%$ of the variance in the early onset ASB measure, $40 \%$ of the variance in the adult onset ASB measure, and $41 \%$ of the variance in the lifetime ASB measure.

The regression models were estimated a second time using the sex-restricted subsamples. The results for the male only subsample closely aligned with the results of the full sample, with the pedigree risk factor explaining 38\% of the overall variance in early onset ASB, 39\% of the variance in adult onset $\mathrm{ASB}$, and $41 \%$ of the variance in the lifetime ASB measure. The results from the models examining the female only subsample followed the same general pattern, but the proportion of overall variance explained in each ASB measure was greater than the proportion explained in the full sample and the male only subsample. More specifically, the pedigree risk measure explained between $44 \%$ and $49 \%$ of the overall variance in the examined ASB measures within the female only subsample. In addition, the results of Wald's tests revealed that the resulting associations were significantly larger within the female subsample relative to the male subsample.

The second set of columns in Table 3 presents the results of regression models in which each ASB measure was regressed on the pedigree risk measure, in which the factor loadings for first-and second-degree relatives were constrained to equality. The more restricted measure of pedigree risk resulted in slightly attenuated proportions of explained variance within the three ASB measures, with estimates ranging between $37 \%$ (early onset) and $40 \%$ (lifetime) in the full sample. The overall pattern of results ob- served in the male subsample closely followed those observed in the full sample, while the pattern of results for the female subsample revealed larger associations, with the proportion of explained variance ranging between $44 \%$ and $48 \%$. Once again, Wald's tests revealed that the observed associations in the female subsample were significantly greater than those observed in the male subsample.

The final step in the analysis involved estimating the same regression models a third time, but substituting the most restrictive measurement strategy for the pedigree risk factor, in which the factor loadings for first-degree relatives were fixed to 0.50 and the factor loadings for second-degree relatives were fixed to 0.25 . Once again the overall pattern of findings was similar to those observed with other measurement strategies, although the overall proportion of explained variance was attenuated. Within the full sample, the pedigree risk measure explained between $35 \%$ and $37 \%$ of the overall variance in the examined ASB measures. Within the sex-restricted subsamples, a similar pattern emerged with the pedigree risk measure explaining between 37\% and $40 \%$ of the variance in ASB within the male subsample, and between $40 \%$ and $44 \%$ of the variance within the female subsample. While the results from the female subsample consistently revealed greater levels of explained variance, the Wald's tests only revealed a significant difference between the male and female subsamples for the early onset measure and a marginally significant difference for the lifetime ASB measure.

\section{Supplemental Analyses}

In an effort to assess the robustness of the findings reported in the primary analysis, a series of supplemental analyses was also performed. The results of the supplementary analyses are summarized in Table 4 , and more detailed results (e.g., unstandardized and standardized path coefficients, standard errors, and sample sizes) are reported in the online supplement. The first set of analyses attempted to better specify the individual contributions of the firstand second-degree family risk indicators. The primary purpose of this stage of the analysis was to more thoroughly investigate the predictive ability of the proposed extended pedigree risk approach when fewer family risk indicators are available. In line with these objectives, pedigree risk was measured using a series of two-factor CFA models. The same three measurement strategies used in the primary analyses were employed again, except that separate factors for firstand second-degree family risk indicators were created. The regression models described in the primary analysis were then estimated again using the alternative two-factor pedigree risk measures. Importantly, the two factors were included in the equations separately in an effort to assess the independent association (and corresponding proportion of variance explained) between each measure and the examined ASB outcomes. The overall pattern of results indicated that the first-degree pedigree risk factor consistently 
TABLE 3

Models Estimating the Proportion of Variance Explained in Antisocial Behavior

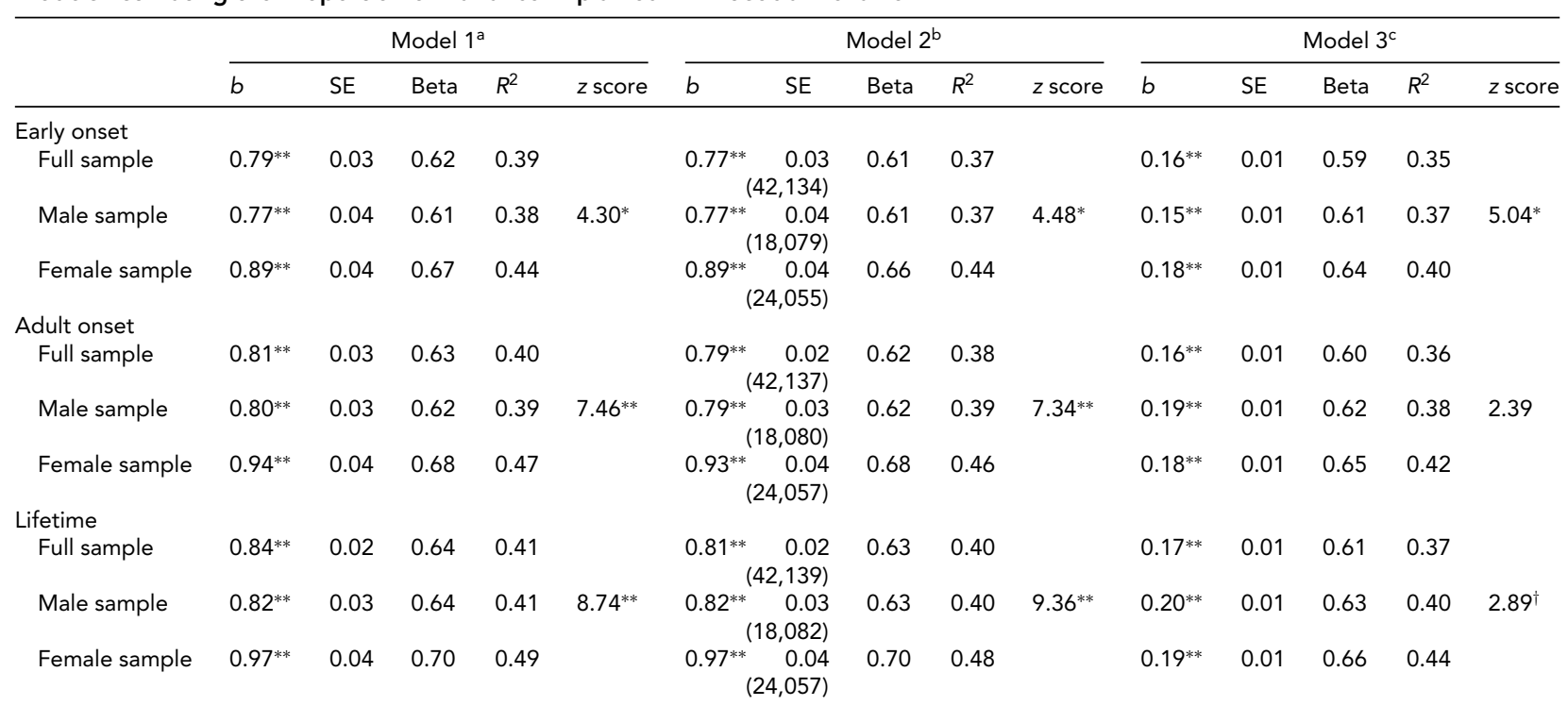

Note: All models were estimated using sample weights and cluster variables. ${ }^{a}$ Family risk indicators allowed to freely load on pedigree risk measure. ${ }^{b}$ Family risk indicator loadings for first- and second-degree relatives constrained to equality. ${ }^{c}$ First-degree family risk indicators constrained to 0.50 and seconddegree family risk indicators constrained to 0.25 . The reported $z$ scores are the results of Wald's tests comparing the path estimate from the male subsample with the path estimate from the female subsample for each examined outcome. Sample sizes presented in parentheses. ${ }^{\dagger} p<.10 ; * p<.05 ;{ }^{* *} p<.01$.

\section{TABLE 4}

Proportion of Variance in Antisocial Behavior Explained in Supplemental Analyses

\begin{tabular}{llll}
\hline & Model 1 & Model $2^{\mathrm{b}}$ & Model 3c \\
\hline Early onset & & & \\
1st degree & 0.43 & 0.43 & 0.38 \\
2nd degree & 0.30 & 0.30 & 0.28 \\
MIMIC & 0.31 & 0.31 & 0.26 \\
Adult onset & & & \\
1st degree & 0.43 & 0.44 & 0.36 \\
2nd degree & 0.31 & 0.32 & 0.29 \\
MIMIC & 0.36 & 0.36 & 0.30 \\
Anytime & & & \\
1st degree & 0.46 & 0.46 & 0.38 \\
2nd degree & 0.32 & 0.32 & 0.30 \\
MIMIC & 0.37 & 0.37 & 0.31 \\
\hline
\end{tabular}

Note: MIMIC = multiple indicator multiple causes. All models were estimated using sample weights and cluster variables. MIMIC models adjust the pedigree risk measure for 23 family risk measures tapping three domains: (1) parental maltreatment (14 items); (2) sexual abuse (4

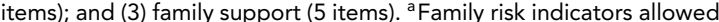
to freely load on pedigree risk measure. ${ }^{b}$ Family risk indicator loadings for first- and second-degree relatives constrained to equality. ${ }^{c}$ Firstdegree family risk indicators constrained to 0.50 and second-degree family risk indicators constrained to 0.25 .

All accompanying coefficients significant at the $p<.01$ level.

explained greater levels of overall variance in ASB relative to the second-degree pedigree risk factor. This pattern of findings was expected since first-degree relatives share a greater proportion of genetic material and are also more likely to share environmental influences with one another compared to second-degree relatives.

Collectively, these findings reveal that a more comprehensive measure of pedigree risk containing information from both first- and second-degree relatives should be favored. In situations in which less information is available, more restrictive measurement strategies (e.g., fixing factor loadings to reflect levels of genetic relatedness) involving first-degree relatives should be favored. These recommendations are largely based on the overall similarity between the results from Model 3 in the supplementary analysis and the results from Models 2 and 3 in the primary analysis.

A second set of supplemental analyses was performed using a series of Multiple Indicator Multiple Causes (MIMIC) models. MIMIC models typically consist of two simultaneously estimated equations within a structural equation modeling (SEM) framework (Jöreskog \& Goldberger, 1975; Muthén, 1989). The first model typically defines one or more latent constructs and is analogous to a traditional CFA model. The second equation consists of a regression model in which the latent factor (or factors) defined in the previous step is regressed on a series of covariates. This second equation effectively removes any variance from the latent factor(s) that can be explained by the included covariates, with the residual variance providing a more precise measure of the intended concept(s).

The MIMIC models estimated at this stage of the analysis consisted of the same three single-factor, latent measures of pedigree risk estimated in the primary analyses, but also included a total of 23 adverse family environmental measures tapping three domains: parental maltreatment, sexual abuse, and family support (see the online supplement for more information). The single-factor pedigree risk measures used in the primary analysis were regressed on all 
23 adverse family environment measures prior to the estimation of the regression models estimating the proportion of variance explained in each of the three ASB measures. As expected, the adjusted pedigree risk measures explained overall lower levels of variance in each of the ASB measures, with estimates ranging between $26 \%$ and $37 \%$. While such attenuated effects may be interpreted as a further isolation of genetic (as opposed to environmental) influences, this pattern of findings may also reflect other, unwarranted processes. More specifically, the employed MIMIC models likely remove variance explained by both environmental and genetic influences. Since at least some of the employed adverse family environment measures are influenced by both genetic and environmental factors (Kendler \& Baker, 2007), removing all of the variance explained by these measures likely artificially deflates the influence of genetic risk. Based on this concern and the overall convergence in findings between the primary and supplemental analyses, it appears that the measurement models presented in the primary analysis should be favored unless there is sufficient theoretical reasoning to employ a MIMIC approach.

\section{Discussion}

Over the past few decades, quantitative genetic studies have effectively illustrated that variation in virtually every measured phenotype can be explained by a combination of genetic and environmental influences (Plomin et al., 2013; Turkheimer, 2000). Based on the overwhelming amount of convergence in these findings, scholars have encouraged researchers to move 'beyond heritability' by utilizing statistical techniques capable of providing a fuller understanding of the etiological development of various behavioral phenotypes (Johnson et al., 2009; McGue et al., 2010; Turkheimer, 2011). The current study aimed to contribute to this literature by proposing a pedigree risk approach that takes into account the intergenerational transmission of ASB across first- and second-degree relatives in a sample of singletons. Using self-reported information on the prevalence of ASB among first- and second-degree family members, the extended pedigree risk approach was proposed and evaluated by examining the overall proportion of explained variance in multiple comprehensive measures of ASB. Importantly, the primary goal of this evaluation was not to simply explain variance, but rather to compare the performance of the created pedigree risk factors to the consistent pattern of findings flowing from previous studies employing twin- or adoption-based research designs.

The results of the employed analyses yielded two main findings that require additional discussion. First, the results of the regression analyses revealed that the employed pedigree risk factor explained between $35 \%$ and $49 \%$ of the variance in the examined ASB measures depending on the subsample examined and the measurement strategy used. These findings are quite similar to the overall pattern of results that emerge from a vast number of studies that employ traditional twin- and adoption-based research designs to decompose variance in ASB (Ferguson, 2010; Mason \& Frick, 1994; Miles \& Carey, 1997; Rhee \& Waldman, 2002). While the overall patterns of findings are similar, the proportion of variance explained by the pedigree risk factor employed in the current study was attenuated, particularly when employing the most restrictive measurement strategy. More specifically, the results of the current study revealed a negative relationship between measurement restriction in the pedigree risk factor and overall variance explained in ASB, wherein the most restricted pedigree risk factor (i.e., factor loadings were constrained to reflect levels of genetic relatedness) resulted in overall lower levels of explained variance in ASB. While only preliminary, we offer two separate explanations for this attenuated effect.

First, such patterns may stem directly from respondents' underestimation of ASBs within their family. More specifically, the employed pedigree risk measure directly relies on two related sources of information: (a) the respondent's knowledge of each of their family members' behaviors; and (b) the accuracy with which such information is reported. Distortions in either source of information would result in changes in the pedigree risk factor. However, it is reasonable to expect that respondents would be more likely to underestimate the prevalence of ASB within family members, particularly second-degree family members, simply because they are not aware of such behaviors. This underreporting would result in an attenuation of the proportion of variance in ASB explained by the resulting pedigree risk measure by artificially deflating scores for respondents who were not aware of some family members' behaviors or who did not accurately report such information. ${ }^{4}$

The second explanation for the attenuated effect of the pedigree risk measure may stem from the models that included more restricted measurement strategies. The purpose of such strategies was two-fold: (1) to construct a pedigree risk measure that adequately reflects additive genetic theory; and (2) to limit the amount of variance in the resulting measure attributable to shared environmental influences. Directly in line with these objectives, one potential interpretation of the observed attenuation in the overall proportion of ASB variance explained by the more restrictive measures of pedigree risk is that such measures better isolate sources of genetic influence. As mentioned previously, even the most restrictive measurement strategy likely fails to fully partial out variance stemming from shared environments. However, previous studies have revealed that shared environments explain a relatively small proportion of the overall variance in ASB (Ferguson, 2010; Mason \& Frick, 1994; Miles \& Carey, 1997; Rhee \& Waldman, 2002), and such influences tend to diminish over the life course (Bergen et al., 2007). Since the NESARC contains a nationally representative sample of U.S. adults, the average age of the sample, 45.24 years old, reflects the average age of 
the target population. Based on these two observations, it seems quite likely that the collective influence of the shared environment on the examined outcomes is minimal.

While the measurement strategy employed in the current study does not allow genetic influences to be fully separated from shared environmental influences, such an application does not compromise the primary objective of the extended pedigree risk approach. Rather than directly estimate the proportion of phenotypic variance attributable to genetic and shared environmental influences, this approach is intended to be used in multivariate, quasi-experimental research designs in an effort to better isolate observed sources of environmental influence on various behavioral phenotypes. The conflation of both genetic and shared environmental influences should not undermine such an objective. Rather, more conventional and commonly used quantitative genetic modeling strategies include controls for both genetic and shared environmental influences in an effort to better isolate potential associations between non-shared environmental influences and phenotypic outcomes. For example, the widely used DeFries-Fulker (DF) equation has been modified to include statistical controls for genetic and shared environmental influences (Rodgers et al., 1994), and the monozygotic twin difference score approach inherently isolates associations between sources of non-shared environmental influence and phenotypic outcomes (Asbury et al., 2003; Burt et al., 2006). Finally, previous studies employing multivariate biometric modeling strategies typically include controls for both genetic and shared environmental influences (e.g., see Harden, 2014). Taken together, these findings indicate that while the extended pedigree risk approach employed in the current study is limited, such an approach can still be used to minimize model misspecification stemming from uncontrolled spuriousness.

The second main finding to emerge from the current study is related to the findings from the sex-restricted models. Regardless of the measurement strategy used, the pedigree risk factor explained more variance in all three of the examined ASB measures for the female-only subsample relative to the male-only subsample, with only two exceptions. When employing the most restrictive pedigree risk measure, the difference between males and females was only marginally significant for the lifetime ASB measure, and was non-significant for adult onset ASB. This pattern of findings directly aligns with previous quantitative genetic studies (Cross et al., 2011; Eley et al., 1999; Jacobson et al., 2002; Schwartz \& Beaver, 2015) and is so pervasive that theoretical models have been proposed to account for greater levels of genetic risk on ASB for females relative to males (Campbell, 2002; Vaske et al., 2011). While still only preliminary, these findings provide further confidence in the extended pedigree risk approach.

In addition to those discussed above, the current study possessed three limitations. First, and perhaps most importantly, the extended pedigree risk approach is expected to result in a more crude estimation of genetic and environmental influence and is not intended to be a replacement for more precise estimates, such as those estimated using twinbased methodologies. Rather, this alternative measurement strategy is intended for use in situations when the only other alternative is to estimate statistical models that are almost certainly misspecified due to genetic confounding (e.g., see Armour \& Haynie, 2007). While more traditional methods of accounting for genetic confounding should be used whenever possible, this alternative modeling strategy seems promising in situations when data restrictions prohibit such approaches. Clearly, replication and additional research are required to further assess the validity and reliability of this alternative strategy, but the results of the current study provide preliminary support.

Second, since the NESARC does not contain twin or sibling pairs, it was not possible to directly compare the proportion of variance explained by the pedigree risk factors to the proportion explained using traditional quantitative genetic modeling strategies. Due to this limitation, we were required to compare the findings from the current study with the overall pattern of findings within the existing literature. Fortunately, and as previously discussed, this literature is vast and the findings flowing from it seem to converge nicely, providing a reasonable criterion with which comparisons can be made. Moving forward, future research would benefit from exploring samples that contain both twins/sibling pairs and self-reported family behavior measures in an effort to more directly compare the validity of the proposed approach. Third, the extended pedigree risk approach presented in the current study is limited to examining associations between non-shared environments and phenotypic outcomes. Since shared environmental influences cannot be directly modeled and are either included in the pedigree risk measure or directly excluded from the model, studies attempting to examine associations between sources of the shared environment and phenotypic outcomes would be required to employ an alternative analytic strategy.

The current study proposed a pedigree risk approach for use with samples that do not contain the necessary information to estimate genetically informed models using more traditional quantitative genetic methods. The results largely suggest that this approach offers a feasible alternative modeling strategy that allows for the subsequent application of a wide range of statistical techniques. While future and ongoing data collection projects would certainly benefit from the oversampling of twin or sibling pairs, this alternative approach requires far fewer data restrictions than more traditional quantitative genetic techniques, allowing for the use of a much larger range of potential samples to further explore the underlying etiology of various behavioral outcomes. This expansion of available data is critically important for disciplines in which samples containing twins and genome-wide information remain relatively rare, 
such as criminology and sociology. Importantly, many of these same disciplines have only recently begun to fully appreciate the importance of employing genetically sensitive research designs and the pervasiveness of model misspecification stemming from genetic confounding (Barnes et al., 2014; Connolly \& Beaver, 2014; Coyne et al., 2013; Schwartz et al., 2014). This alternative modeling strategy provides yet another analytic tool aimed at providing a more comprehensive understanding of the underlying processes which ultimately result in behavioral variation.

\section{Acknowledgments}

The authors wish to thank JC Barnes and Nick Martin for their helpful comments on a previous draft of this study.

\section{Endnotes}

1 Two of the included items (ever cut class and ever stayed out late at night without permission) were asked following the same format, but the age specified in the follow-up questions was 13 instead of 15 .

2 Respondents were asked about each type of relative separately and explicit definitions were provided. For example, when asked about the number of maternal aunts in their family, respondents were asked to report the 'number of their natural mother's full sisters who lived to be 10 years old'.

3 Six items loaded strongly on multiple factors. The majority of these items were rather vague, including lying a lot, doing something that could have resulted in an arrest, and bullying others. All of the analyses performed in the current study were estimated a second time and these items were allowed to cross-load onto multiple factors. The results of these supplementary analyses were virtually identical to the ones reported in the current study.

4 This particular limitation could potentially be addressed by examining an alternative phenotype that: (1) is easily recognizable and salient; and (2) has been investigated by an extensive literature, which has produced largely concordant findings regarding the proportion of variance explained by additive genetic influence. One potential phenotype fitting this description is body mass index (BMI). Unfortunately, the NESARC does not include extended pedigree BMI measures. Due to this limitation, we were unable to investigate the performance of the proposed pedigree risk measure in the examination of BMI.

\section{Supplementary Material}

To view supplementary material for this article, please visit http://dx.doi.org/10.1017/thg.2015.68.

\section{References}

Armour, S., \& Haynie, D. L. (2007). Adolescent sexual debut and later delinquency. Journal of Youth and Adolescence, 36, 141-152.

Asbury, K., Dunn, J. F., Pike, A., \& Plomin, R. (2003). Nonshared environmental influences on individual differences in early behavioral development: A monozygotic twin difference study. Child Development, 74, 933-943.

Baker, L. A., Jacobson, K. C., Raine, A., Lozano, D. I., \& Bezdjian, S. (2007). Genetic and environmental bases of childhood antisocial behavior: A multi-informant twin study. Journal of Abnormal Psychology, 116, 219-235.

Barnes, J. C., Beaver, K. M., \& Boutwell, B. B. (2011). Examining the genetic underpinnings to Moffitt's developmental taxonomy: A behavioral genetic analysis. Criminology, 49, 923-954.

Barnes, J. C., \& Boutwell, B. B. (2013). A demonstration of the generalizability of twin-based research on antisocial behavior. Behavior Genetics, 43, 120-131.

Barnes, J. C., Wright, J. P., Boutwell, B. B., Schwartz, J. A., Connolly, E. J., Nedelec, J. L., \& Beaver, K. M. (2014). Demonstrating the validity of twin research in criminology. Criminology, 52, 588-626.

Beaver, K. M., Schwartz, J. A., Connolly, E.J., Nedelec, J. L., AlGhamdi, M. S., \& Kobeisy, A. N. (2013). The genetic and environmental architecture to the stability of IQ: Results from two independent samples of kinship pairs. Intelligence, 41, 428-438.

Bergen, S. E., Gardner, C. O., \& Kendler, K. S. (2007). Agerelated changes in heritability of behavioral phenotypes over adolescence and young adulthood: A meta-analysis. Twin Research and Human Genetics, 10, 423-433.

Burt, S. A., McGue, M., Iacono, W. G., \& Krueger, R. F. (2006). Differential parent-child relationships and adolescent externalizing symptoms: Cross-lagged analyses within a monozygotic twin differences design. Developmental Psychology, 42, 1289-1298.

Byrne, B. M. (2012). Structural equation modeling with Mplus: Basic concepts, applications, and programming. New York, NY: Taylor \& Francis.

Campbell, A. (2002). A mind of her own: the evolutionary psychology of women. London: Oxford University Press.

Carey, G. (2003). Human genetics for the social sciences. Thousand Oaks, CA: Sage.

Connolly, E. J., \& Beaver, K. M. (2014). Examining the genetic and environmental influences on self-control and delinquency: Results from a genetically informative analysis of sibling pairs. Journal of Interpersonal Violence, 29, 707735.

Coyne, C. A., Fontaine, N. M., Långström, N., Lichtenstein, P., \& D'Onofrio, B. M. (2013). Teenage childbirth and young adult criminal convictions: A quasi-experimental study of criminal outcomes for teenage mothers. Journal of Criminal Justice, 41, 318-323.

Cross, C. P., Copping, L. T., \& Campbell, A. (2011). Sex differences in impulsivity: A meta-analysis. Psychological Bulletin, 137, 97-130.

DeFries, J. C., \& Fulker, D. W. (1985). Multiple regression analysis of twin data. Behavior Genetics, 15, 467-473.

Eley, T. C., Lichtenstein, P., \& Stevenson, J. (1999). Sex differences in the etiology of aggressive and nonaggressive antisocial behavior: Results from two twin studies. Child Development, 70, 155-168. 
Ellis, L., \& Walsh, A. (2000). Criminology: A global perspective. Boston, MA: Allyn \& Bacon.

Ferguson, C. J. (2010). Genetic contributions to antisocial personality and behavior: A meta-analytic review from an evolutionary perspective. The Journal of Social Psychology, 150, $160-180$.

Grant, B. F., Dawson, D. A., \& Hasin, D. S. (2001). The alcohol use disorder and associated disabilities interview schedule - DSM-IV version. Bethesda, MD: National Institute on Alcohol Abuse and Alcoholism.

Grant, B. F., Kaplan, K., Shepard, J., \& Moore, T. (2003). Source and accuracy statement for the wave 1 of the 2001-2002 national epidemiologic survey on alcohol and related conditions. Bethesda, MD: National Institute on Alcohol Abuse and Alcoholism.

Graubard, B. I., \& Sirken, M. G. (2011). Estimating sibling recurrence risk in population sample surveys. Human Heredity, 76, 18-27.

Guo, S. W. (1998). Inflation of sibling recurrence-risk ratio, due to ascertainment bias and/or overreporting. The American Journal of Human Genetics, 63, 252-258.

Haberstick, B. C., Smolen, A., Stetler, G. L., Tabor, J. W., Roy, T., Casey, H. R., ... Harris, K. M. (2014). Simple sequence repeats in the national longitudinal study of adolescent health: An ethnically diverse resource for genetic analysis of health and behavior. Behavior Genetics, 44, 487497.

Harden, K. P. (2014). Genetic influences on adolescent sexual behavior: Why genes matter for environmentally oriented researchers. Psychological Bulletin, 140, 434-465.

Harden, K. P., Mendle, J., Hill, J. E., Turkheimer, E., \& Emery, R. E. (2008). Rethinking timing of first sex and delinquency. Journal of Youth and Adolescence, 37, 373-385.

Harris, J. R. (2009). The nurture assumption: Why children turn out the way they do (2nd ed.). New York, NY: Free Press.

Hu, L. T., \& Bentler, P. M. (1999). Cutoff criteria for fit indexes in covariance structure analysis: Conventional criteria versus new alternatives. Structural Equation Modeling: $A$ Multidisciplinary Journal, 6, 1-55.

Hur, Y., \& Craig, J. M. (2013). Twin registries worldwide: An important resource for scientific research. Twin Research and Human Genetics, 16, 1-12.

Jacobson, K. C., Prescott, C. A., \& Kendler, K. S. (2002). Sex differences in the genetic and environmental influences on the development of antisocial behavior. Development and Psychopathology, 14, 395-416.

Johnson, W., \& Krueger, R. F. (2005). Genetic effects on physical health: Lower at higher income levels. Behavior Genetics, $35,579-590$.

Johnson, W., Turkheimer, E., Gottesman, I. I., \& Bouchard, T. J. (2009). Beyond heritability twin studies in behavioral research. Current Directions in Psychological Science, 18, 217-220.

Jöreskog, K. G., \& Goldberger, A. S. (1975). Estimation of a model with multiple indicators and multiple causes of a single latent variable. Journal of the American Statistical Association, 70, 631-639.
Kendler, K. S., \& Baker, J. H. (2007). Genetic influences on measures of the environment: A systematic review. Psychological Medicine, 37, 615-626.

Leonard, E. (1995). Theoretical criminology and gender. In B. Price \& N. Sokoloff (Eds.), The criminal justice system and women: Offenders, victims, and workers (pp. 54-70). New York, NY: McGraw-Hill.

Maes, H. H., Neale, M. C., \& Eaves, L. J. (1997). Genetic and environmental factors in relative body weight and human adiposity. Behavior Genetics, 27, 325-351.

Martel, M. M. (2013). Sexual selection and sex differences in the prevalence of childhood externalizing and adolescent internalizing disorders. Psychological Bulletin, 139, 12211259.

Mason, D. A., \& Frick, P. J. (1994). The heritability of antisocial behavior: A meta-analysis of twin and adoption studies. Journal of Psychopathology and Behavioral Assessment, 16, 301-323.

McGue, M., \& Christensen, K. (2003). The heritability of depression symptoms in elderly Danish twins: Occasionspecific versus general effects. Behavior Genetics, 33, 8393.

McGue, M., Osler, M., \& Christensen, K. (2010). Causal inference and observational research: The utility of twins. Perspectives on Psychological Science, 5, 546-556.

McGuffin, P., Rijsdijk, F., Andrew, M., Sham, P., Katz, R., \& Cardno, A. (2003). The heritability of bipolar affective disorder and the genetic relationship to unipolar depression. Archives of General Psychiatry, 60, 497-502.

Miles, D. R., \& Carey, G. (1997). Genetic and environmental architecture on human aggression. Journal of Personality and Social Psychology, 72, 207-217.

Moffitt, T. E. (1993). Adolescence-limited and life-coursepersistent antisocial behavior: A developmental taxonomy. Psychological Review, 100, 674-701.

Moffitt, T. E. (2005). Genetic and environmental influences on antisocial behaviors: Evidence from behavioral-genetic research. Advances in Genetics, 55, 41-104.

Muthén, B. O. (1989). Latent variable modeling in heterogeneous populations. Psychometrika, 54, 557-585.

Muthén, L. M., \& Muthén, B. O. (2013). Mplus user's guide (7th ed.). Los Angeles, CA: Muthén \& Muthén.

Neale, M. C., \& Cardon, L. R. (1992). Methodology for genetic studies of twins and families. Dordrecht, the Netherlands: Kluwer Academic.

Plomin, R., DeFries, J. C., Knopik, V. S., \& Neiderhiser, J. M. (2013). Behavioral genetics (6th ed.). New York, NY: Worth Publishers.

Purcell, S., Neale, B., Todd-Brown, K., Thomas, L., Ferreira, M. A., Bender, D., ... Sham, P. C. (2007). PLINK: A tool set for whole-genome association and population-based linkage analyses. The American Journal of Human Genetics, $81,559-575$.

Rhee, S. H., \& Waldman, I. D. (2002). Genetic and environmental influences on antisocial behavior: A meta-analysis of twin and adoption studies. Psychological Bulletin, 128, $490-529$. 
Risch, N. (1990a). Linkage strategies for genetically complex traits. I. Multilocus models. American Journal of Human Genetics, 46, 222-228.

Risch, N. (1990b). Linkage strategies for genetically complex traits. II. The power of affected relative pairs. American Journal of Human Genetics, 46, 229-241.

Risch, N. (1990c). Linkage strategies for genetically complex traits. III. The effect of marker polymorphism on analysis of affected relative pairs. American Journal of Human Genetics, 46, 242-253.

Rodgers, J. L., Rowe, D. C., \& Li, C. (1994). Beyond nature versus nurture: DF analysis of nonshared influences on problem behaviors. Developmental Psychology, 30, 374-384.

Schwartz, J. A., \& Beaver, K. M. (2015). A partial test of Moffitt's developmental taxonomy: Examining the role of genetic risk. Justice Quarterly, 32, 768-791.

Schwartz, J. A., Rowland, M. W., \& Beaver, K. M. (2014). A genetically informed test of cholesterol levels and self-control, depressive symptoms, antisocial behavior, and neuroticism. Journal of Affective Disorders, 164, 139-147.
Trzaskowski, M., Eley, T. C., Davis, O. S., Doherty, S. J., Hanscombe, K. B., Meaburn, E. L., ... Plomin, R. (2013). First genome-wide association study on anxiety-related behaviours in childhood. PloS One, 8, e58676.

Turkheimer, E. (2000). Three laws of behavior genetics and what they mean. Current Directions in Psychological Science, 9, 160-164.

Turkheimer, E. (2011). Commentary: Variation and causation in the environment and genome. International Journal of Epidemiology, 40, 598-601.

Vaske, J., Wright, J. P., Boisvert, D., \& Beaver, K. M. (2011). Gender, genetic risk, and criminal behavior. Psychiatry Research, 185, 376-381.

Wallace, C., Clayton, D., \& Fine, P. (2003). Estimating the relative recurrence risk ratio for leprosy in Karonga District, Malawi. Leprosy Review, 74, 133-140.

Yang, J., Lee, S. H., Goddard, M. E., \& Visscher, P. M. (2011). GCTA: A tool for genome-wide complex trait analysis. American Journal of Human Genetics, 88, 7682. 